Summary of Effects of Chemical Agents

\title{
1) Ocular
}

\begin{tabular}{|c|c|c|c|c|c|c|}
\hline Agent & Symbol & Odour & $\begin{array}{l}\text { Mechanism of } \\
\text { action }\end{array}$ & \begin{tabular}{|l|} 
Eyes \\
(pupils)
\end{tabular} & \begin{tabular}{|l|} 
Eyes \\
(conjunctivae)
\end{tabular} & Rest of eye \\
\hline $\begin{array}{l}\text { Tabun } \\
\text { Sarin } \\
\text { Soman } \\
\text { GF } \\
\text { VX }\end{array}$ & $\begin{array}{l}\text { GA } \\
\text { GB } \\
\text { GD } \\
\text { GF } \\
\text { VX }\end{array}$ & $\begin{array}{l}\text { None or faint } \\
\text { sweetish, fruity } \\
\text { or paint-like } \\
\text { None }\end{array}$ & $\begin{array}{l}\text { Anticholinesterase } \\
\text { agents }\end{array}$ & Miosis & Redness & $\begin{array}{l}\text { Pain, especially on } \\
\text { focusing dimness } \\
\text { of vision, headache, } \\
\text { lachrymation }\end{array}$ \\
\hline $\begin{array}{l}\text { Mustard } \\
\text { and } \\
\text { nitrogen } \\
\text { mustard }\end{array}$ & $\begin{array}{l}\mathrm{H} \\
\mathrm{HD} \\
\mathrm{HN}\end{array}$ & $\begin{array}{l}\text { Garlic or } \\
\text { horseradish, } \\
\text { irritating. } \\
\text { None or fishy, } \\
\text { irritating }\end{array}$ & \begin{tabular}{|l|} 
Vesicants. Bone \\
marrow depressant. \\
Alkylating agents, \\
damages DNA
\end{tabular} & Mydriasis & $\begin{array}{l}\text { Redness, } \\
\text { oedema, } \\
\text { irritation, } \\
\text { gritty } \\
\text { pain }\end{array}$ & $\begin{array}{l}\text { Oedema of lids, pain, } \\
\text { blepharospasm, } \\
\text { photophobia, } \\
\text { lachrymation, corneal } \\
\text { ulceration and } \\
\text { possibly scarring }\end{array}$ \\
\hline $\begin{array}{l}\text { Lewisite and } \\
\text { other arsenical } \\
\text { vesicants }\end{array}$ & $\mathrm{L}$ & $\begin{array}{l}\text { Fruity to geranium } \\
\text {-like. Irritating }\end{array}$ & $\begin{array}{l}\text { Vesicants. Arsenical } \\
\text { poisons }\end{array}$ & & $\begin{array}{l}\text { Prompt } \\
\text { redness, } \\
\text { oedema, } \\
\text { irritation }\end{array}$ & $\begin{array}{l}\text { Immediate burning } \\
\text { sensation, iritis, } \\
\text { corneal injury }\end{array}$ \\
\hline $\begin{array}{l}\text { Mustard/ } \\
\text { Lewisite } \\
\text { mixture }\end{array}$ & $\mathrm{HL}$ & Garlic-like & $\begin{array}{l}\text { Like Lewisite and } \\
\text { mustard }\end{array}$ & $\begin{array}{l}\text { Like HD, } \\
\text { HN and L }\end{array}$ & $\begin{array}{l}\text { Like HD, } \\
\mathrm{HN} \text { and L }\end{array}$ & Like $\mathrm{HD}, \mathrm{HN}$ and $\mathrm{L}$ \\
\hline $\begin{array}{l}\text { Phosgene } \\
\text { oxime }\end{array}$ & CX & $\begin{array}{l}\text { Unpleasant and } \\
\text { irritating }\end{array}$ & Powerful vesicant & & $\begin{array}{l}\text { Volently } \\
\text { irritating, } \\
\text { redness, } \\
\text { oedema }\end{array}$ & $\begin{array}{l}\text { Corneal injury with } \\
\text { blindness } \\
\text { lachrymation }\end{array}$ \\
\hline Phosgene & CG & $\begin{array}{l}\text { Green com, grass } \\
\text { or new-mown hay }\end{array}$ & $\begin{array}{l}\text { Lung damaging } \\
\text { agent }\end{array}$ & & Irritation & $\begin{array}{l}\text { Lachrymation (after } \\
\text { respiratory symptoms) }\end{array}$ \\
\hline $\begin{array}{l}\text { Hydrogen } \\
\text { cyanide }\end{array}$ & AC & $\begin{array}{l}\text { Faint bitter } \\
\text { almonds }\end{array}$ & $\begin{array}{l}\text { Interferes with } \\
\text { oxygen utilisation } \\
\text { at cellular level }\end{array}$ & & & \\
\hline $\begin{array}{l}\text { Cyanogen } \\
\text { cyanide }\end{array}$ & CK & Very irritating & $\begin{array}{l}\text { Like hydrogen } \\
\text { cyanide, lung irritant }\end{array}$ & & Irritation & Lachrymation \\
\hline $\begin{array}{l}\text { Vomiting } \\
\text { agents }\end{array}$ & $\begin{array}{l}\mathrm{DM} \\
\mathrm{DA} \\
\mathrm{DC}\end{array}$ & $\begin{array}{l}\text { Burning, fireworks, } \\
\text { v irritating }\end{array}$ & $\begin{array}{l}\text { Local irritant, } \\
\text { induces vomiting }\end{array}$ & & Irritation & Lachrymation \\
\hline \multirow[t]{2}{*}{ Irritant agents } & $\mathrm{CN}$ & Irritating & Local irritant & & $\begin{array}{l}\text { Redness, } \\
\text { irritation }\end{array}$ & $\begin{array}{l}\text { Pain, blephoraspasm, } \\
\text { profuse lachrymation, } \\
\text { photophobia }\end{array}$ \\
\hline & $\begin{array}{l}\mathrm{CS} \\
\mathrm{CR}\end{array}$ & $\begin{array}{l}\text { Very irritating, } \\
\text { pungent, pepper- } \\
\text { like }\end{array}$ & Local irritant & & $\begin{array}{l}\text { Intense } \\
\text { irritation }\end{array}$ & $\begin{array}{l}\text { Pain, blephoraspasm, } \\
\text { lachrymation, } \\
\text { photophobia }\end{array}$ \\
\hline \multirow[t]{2}{*}{$\begin{array}{l}\text { Incapacitating } \\
\text { agents }\end{array}$} & $\mathrm{BZ}$ & None & Anticholinergic & Myadris & & Blurred vision \\
\hline & LSD & None & Psychomimetic & Mydriasis & & \\
\hline
\end{tabular}


2) Nose and Throat; respiratory; skin; GI tract, cardiovascular

\begin{tabular}{|c|c|c|c|c|c|}
\hline Symbol & \begin{tabular}{|l|} 
Nose and \\
Throat
\end{tabular} & Respiratory & Skin & GI tract & \begin{tabular}{|l|} 
Cardiovascular \\
system
\end{tabular} \\
\hline $\begin{array}{l}\text { GA } \\
\text { GB } \\
\text { GD } \\
\text { GF } \\
\text { VX }\end{array}$ & $\begin{array}{l}\text { Increased } \\
\text { salivation, } \\
\text { rhinorrhoea }\end{array}$ & $\begin{array}{l}\text { Tightness in the chest, } \\
\text { bronchoconstriction, } \\
\text { occasional wheezing, } \\
\text { increased bronchial } \\
\text { secretion, cough, } \\
\text { dyspnoea, substernal } \\
\text { tightness }\end{array}$ & $\begin{array}{l}\text { Sweating, pallor } \\
\text { then cyanosis }\end{array}$ & $\begin{array}{l}\text { Salivation, anorexia, } \\
\text { nausea, vomiting, } \\
\text { abdominal cramps, } \\
\text { epigastric tightness, } \\
\text { heartburn, eruction, } \\
\text { diarrhoea, tesmus, } \\
\text { involuntary } \\
\text { defecation }\end{array}$ & $\begin{array}{l}\text { Occasionally early } \\
\text { transient } \\
\text { tachycardia } \\
\text { and/or } \\
\text { hypotension } \\
\text { followed by } \\
\text { bradycardia } \\
\text { and hypotension }\end{array}$ \\
\hline $\begin{array}{l}\mathrm{H} \\
\mathrm{HD} \\
\mathrm{HN}\end{array}$ & $\begin{array}{l}\text { Swelling } \\
\text { irritation, } \\
\text { ulceration, } \\
\text { discharge, } \\
\text { occasional } \\
\text { oedema } \\
\text { of larynx }\end{array}$ & $\begin{array}{l}\text { Slowly developing } \\
\text { irritation, hoarseness, } \\
\text { aphonia, cough, } \\
\text { tightness, dyspnoea, } \\
\text { rales. Pneumonia, } \\
\text { fever, pulmonary } \\
\text { oedema in severe } \\
\text { cases. Risk of } \\
\text { secondary infection }\end{array}$ & $\begin{array}{l}\text { No immediate signs. } \\
\text { After minutes to } \\
\text { hours, redness and } \\
\text { burning. Several } \\
\text { hours later necrosis, } \\
\text { generally limited to } \\
\text { epidermis. Delayed } \\
\text { hyper-and hypo- } \\
\text { pigmentation. } \\
\text { Moist areas affected } \\
\text { most. Risk of } \\
\text { secondary infection. }\end{array}$ & $\begin{array}{l}\text { Pain, nausea, } \\
\text { vomiting, diarrhoea }\end{array}$ & $\begin{array}{l}\text { Shock after } \\
\text { severe exposure }\end{array}$ \\
\hline $\mathrm{L}$ & $\begin{array}{l}\text { Prompt } \\
\text { irritation }\end{array}$ & $\begin{array}{l}\text { Rapid irritation, } \\
\text { hoarseness, aphonia, } \\
\text { cough, pneumonia, } \\
\text { fever, pulmonary } \\
\text { oedema, pleural } \\
\text { effusion in severe } \\
\text { cases }\end{array}$ & $\begin{array}{l}\text { Prompt burning. } \\
\text { Red within } 30 \text { mins. } \\
\text { Blisters on } 1 \text { st or } \\
\text { 2nd day. Pain worse } \\
\text { and necrosis deeper } \\
\text { than H }\end{array}$ & $\begin{array}{l}\text { Diarrhoea, nausea, } \\
\text { vomiting, hepatic } \\
\text { failure }\end{array}$ & \begin{tabular}{|l|} 
Shock after \\
severe exposure. \\
Haemolytic \\
anaemia, haemo- \\
concentration
\end{tabular} \\
\hline $\mathrm{HL}$ & $\begin{array}{l}\text { Swelling } \\
\text { irritation, } \\
\text { ulceration, } \\
\text { discharge, } \\
\text { occasional } \\
\text { oedema } \\
\text { of larynx }\end{array}$ & $\begin{array}{l}\text { Slowly developing } \\
\text { irritation, hoarseness, } \\
\text { aphonia, cough, } \\
\text { tightness, dyspnoea, } \\
\text { rales. Pneumonia, } \\
\text { fever, pulmonary } \\
\text { oedema in severe } \\
\text { cases. Risk of } \\
\text { secondary infection }\end{array}$ & $\begin{array}{l}\text { No immediate signs. } \\
\text { After minutes to } \\
\text { hours, redness and } \\
\text { burning. Several } \\
\text { hours later necrosis, } \\
\text { generally limited to } \\
\text { epidermis. Delayed } \\
\text { hyper-and hypo- } \\
\text { pigmentation. } \\
\text { Moist areas affected } \\
\text { most. Risk of } \\
\text { secondary infection. }\end{array}$ & $\begin{array}{l}\text { Pain, nausea, } \\
\text { vomiting, diarrhoea }\end{array}$ & $\begin{array}{l}\text { Shock after } \\
\text { severe exposure }\end{array}$ \\
\hline $\mathrm{CX}$ & $\begin{array}{l}\text { Very } \\
\text { irritating } \\
\text { to mucous } \\
\text { membranes }\end{array}$ & $\begin{array}{l}\text { Rapid irritation and } \\
\text { coughing. Later } \\
\text { pulmonary oedema }\end{array}$ & $\begin{array}{l}\text { Immediate severe } \\
\text { irritation and intense } \\
\text { pain. Within } 1 \mathrm{~min} \\
\text { the affected area } \\
\text { turns white, } \\
\text { surrounded by } \\
\text { erythema. Swollen } \\
\text { within } 1 \mathrm{hr} \text {; blistered } \\
\text { after } 24 \mathrm{hrs} \text {. } \\
\text { Necrosis may occur. } \\
\text { Long recovery }-1-3 \\
\text { months. }\end{array}$ & & \\
\hline CG & Irritation & $\begin{array}{l}\text { Coughing, choking, } \\
\text { chest tightness on } \\
\text { exposure. Latent } \\
\text { period, then } \\
\text { pulmonary oedema, } \\
\text { dyspnoea, frothy } \\
\text { sputum, rales, } \\
\text { pneumonia and fever }\end{array}$ & $\begin{array}{l}\text { Possible cyanosis } \\
\text { following pulmonary } \\
\text { oedema }\end{array}$ & $\begin{array}{l}\text { Nausea, occasional } \\
\text { vomiting after } \\
\text { respiratory } \\
\text { symptoms }\end{array}$ & $\begin{array}{l}\text { Shock after severe } \\
\text { exposure } \\
\text { hypotension } \\
\text { and tachycardia }\end{array}$ \\
\hline AC & & $\begin{array}{l}\text { Deep respiration } \\
\text { followed rapidly by } \\
\text { dyspnoea, gasping } \\
\text { then cessation of } \\
\text { respiration }\end{array}$ & $\begin{array}{l}\text { Initially pinker than } \\
\text { usual;may change to } \\
\text { cyanosis }\end{array}$ & Nausea & $\begin{array}{l}\text { Profound } \\
\text { hypotension }\end{array}$ \\
\hline CK & Irritation & $\begin{array}{l}\text { Irritation, cough, } \\
\text { choking, dyspnoea; } \\
\text { pulmonary oedema } \\
\text { can be rapid }\end{array}$ & & $\begin{array}{l}\text { Like hydrogen } \\
\text { cyanide }\end{array}$ & \\
\hline $\begin{array}{l}\mathrm{DM} \\
\mathrm{DA} \\
\mathrm{DC}\end{array}$ & $\begin{array}{l}\text { Pain, } \\
\text { rhinorrhoea } \\
\text { tightness, } \\
\text { sneezing }\end{array}$ & $\begin{array}{l}\text { Tightness and pain, } \\
\text { uncontrollable } \\
\text { coughing }\end{array}$ & $\begin{array}{l}\text { Stinging, (especially } \\
\text { of face) occasional } \\
\text { dermatiitis }\end{array}$ & $\begin{array}{l}\text { Salivation, nausea, } \\
\text { vomiting }\end{array}$ & \\
\hline
\end{tabular}




\begin{tabular}{|c|c|c|c|c|c|}
\hline Symbol & $\begin{array}{l}\text { Nose and } \\
\text { Throat }\end{array}$ & Respiratory & Skin & GI tract & $\begin{array}{l}\text { Cardiovascular } \\
\text { system }\end{array}$ \\
\hline $\mathrm{CN}$ & $\begin{array}{l}\text { Irritation, } \\
\text { burning }\end{array}$ & $\begin{array}{l}\text { Tightness and } \\
\text { irritation if } \\
\text { concentration is high }\end{array}$ & $\begin{array}{l}\text { Stinging, (especially } \\
\text { of face) occasional } \\
\text { dermatitis, may } \\
\text { blister }\end{array}$ & Occasional vomiting & \\
\hline $\begin{array}{l}\text { CS } \\
\text { CR }\end{array}$ & $\begin{array}{l}\text { Irritation, } \\
\text { burning, } \\
\text { tightness }\end{array}$ & $\begin{array}{l}\text { Tightness in chest and } \\
\text { difficulty breathing }\end{array}$ & $\begin{array}{l}\text { Stinging, occasional, } \\
\text { dermatitis, may } \\
\text { blister }\end{array}$ & Nausea and vomiting & \\
\hline $\mathrm{BZ}$ & $\begin{array}{l}\text { Extreme } \\
\text { dryness }\end{array}$ & & Dry, flushed & Constipation & $\begin{array}{l}\text { Tachycardia, } \\
\text { elevated blood } \\
\text { pressure }\end{array}$ \\
\hline LSD & & $\begin{array}{l}\text { Sweaty palms, cold } \\
\text { extremities }\end{array}$ & & & Tachycardia \\
\hline
\end{tabular}

\section{3) Genitourinary; CNS; Miscellaneous; Treatment}

\begin{tabular}{|c|c|c|c|c|}
\hline Symbol & GU system & CNS & Other & Treatment \\
\hline $\begin{array}{l}\text { GA } \\
\text { GB } \\
\text { GD } \\
\text { GF } \\
\text { VX }\end{array}$ & $\begin{array}{l}\text { Frequent } \\
\text { micturition, } \\
\text { urinary } \\
\text { incontinence }\end{array}$ & $\begin{array}{l}\text { Apprehension, giddiness, } \\
\text { insomnia, headache, } \\
\text { drowsiness, difficulty } \\
\text { concentrating, poor } \\
\text { memory, confusion, } \\
\text { slurred speech, ataxia, } \\
\text { weakness, coma with } \\
\text { areflexia, Cheyne- } \\
\text { Stokes respiration, } \\
\text { convulsions }\end{array}$ & $\begin{array}{l}\text { Fasciculations, easy } \\
\text { fatigue, cramps, } \\
\text { weakness (including } \\
\text { respiratory muscles) } \\
\text { paralysis }\end{array}$ & $\begin{array}{l}\text { Pre-treatment with pyridostigmine. } \\
\text { Post exposure therapy: } \\
\text { a. Anticholinergic blockage eg. } \\
\text { atropine } \\
\text { b. Enzyme reactivation - oximes } \\
\text { c. Anticonvulsant eg diazepam } \\
\text { d. Assisted ventilation } \\
\text { e. Suction for respiratory secretions }\end{array}$ \\
\hline $\begin{array}{l}\mathrm{H} \\
\mathrm{HD} \\
\mathrm{HN}\end{array}$ & & Anxiety, depression & $\begin{array}{l}\text { Late depression of } \\
\text { bone marrow, } \\
\text { malaise and } \\
\text { prostration }\end{array}$ & $\begin{array}{l}\text { Eyes: antibiotics, cyclopegics and } \\
\text { systemic analgesia. Skin: local } \\
\text { dressings and antibiotics for } \\
\text { infection. Antibiotics for respiratory } \\
\text { infection. IV fluids }\end{array}$ \\
\hline $\mathrm{L}$ & Renal failure & Anxiety, depression & $\begin{array}{l}\text { Systemic arsenic } \\
\text { poisoning }\end{array}$ & $\begin{array}{l}\text { Like sulphur and nitrogen, mustards. } \\
\text { BAL in oil IM for systemic chelation. } \\
\text { BAL ointment for eyes and skin }\end{array}$ \\
\hline $\mathrm{HL}$ & & Anxiety, depression & & $\begin{array}{l}\text { Like sulphur mustard, nitrogen } \\
\text { mustard and Lewisite }\end{array}$ \\
\hline $\mathrm{CX}$ & & Anxiety, depression & & $\begin{array}{l}\text { Apply dressings of sodium } \\
\text { bicarbonate. Systemic anagesics. } \\
\text { Treat as any other skin lesions }\end{array}$ \\
\hline CG & & Anxiety, depression & & $\begin{array}{l}\text { Sorticosteroids IV and inhalation } \\
\text { promptly may be life-saving. Rest, } \\
\text { oxygen, antibiotics }\end{array}$ \\
\hline $\mathrm{AC}$ & & $\begin{array}{l}\text { May have initial excitation; } \\
\text { then depression, giddiness, } \\
\text { headache, irrational } \\
\text { behaviour, ataxia, } \\
\text { convulsions or coma }\end{array}$ & & $\begin{array}{l}\text { Drugs binding cyanide: } \\
\text { a. Methaemoglobin formers; } \\
\text { nitrates or DMAP } \\
\text { b. Scavengers; dicobalt edetate } \\
\text { and hydroxocobalamin } \\
\text { c. Provision of S-groups; } \\
\text { thiosulphate, assisted vent, } \mathrm{O}^{2}\end{array}$ \\
\hline CK & & & & Like hydrogen cyanide and phosgene \\
\hline $\begin{array}{l}\mathrm{DM} \\
\mathrm{DA} \\
\mathrm{DC}\end{array}$ & & $\begin{array}{l}\text { Severe headache, } \\
\text { mental depression }\end{array}$ & $\begin{array}{l}\text { May cause desire to } \\
\text { remove respirator }\end{array}$ & $\begin{array}{l}\text { Wear mask in spite of symptoms. } \\
\text { Spontaneous improvement }\end{array}$ \\
\hline $\begin{array}{l}\mathrm{CN} \\
\mathrm{CA}\end{array}$ & & Headache & & $\begin{array}{l}\text { Spontaneous improvement. } \\
\text { Analgesic eye and nose drops if } \\
\text { necessary }\end{array}$ \\
\hline $\begin{array}{l}\text { CS } \\
\text { CR }\end{array}$ & & Headache & & $\begin{array}{l}\text { Symptoms disappear rapidly in } \\
\text { fresh air }\end{array}$ \\
\hline $\mathrm{BZ}$ & $\begin{array}{l}\text { Urgency, } \\
\text { urinary } \\
\text { retention }\end{array}$ & $\begin{array}{l}\text { Headache, giddiness, } \\
\text { drowsiness, disorientation, } \\
\text { hallucinations and } \\
\text { occasional maniacal } \\
\text { behaviour. Ataxia and/or } \\
\text { lack of coordination }\end{array}$ & & $\begin{array}{l}\text { Restraint, cool environment. } \\
\text { Physostigmine. Treatment may be } \\
\text { required over several days }\end{array}$ \\
\hline LSD & & $\begin{array}{l}\text { Mental excitation, } \\
\text { poor concentration, } \\
\text { tremor indecisiveness, } \\
\text { inability to act in a } \\
\text { sustained or purposeful } \\
\text { manner. Hallucinations }\end{array}$ & Pyrexia & $\begin{array}{l}\text { Reassurance, restraint, prompt } \\
\text { evacuation, diazepam }\end{array}$ \\
\hline
\end{tabular}

